International Journal of Pure and Applied Mathematics

Volume 113 No. 1 2017, 151-165

ISSN: 1311-8080 (printed version); ISSN: 1314-3395 (on-line version)

url: http://www.ijpam.eu

doi: 10.12732/ijpam.v113i1.14

\title{
THE ANALYTICAL SOLUTION OF THE PROBLEM OF THE PURE BEND FOR SHELL MODEL OF THE THIN-WALLED BEAM WITH RECTANGULAR CROSS SECTION
}

\author{
Ilya V. Kudryavtsev ${ }^{1}$, Olga B. Gotseluk ${ }^{2}$, \\ Aleksandr E. Mityaev ${ }^{3}$, Vadim G. Demin ${ }^{4}$ \\ 1,2,3,4 Siberian Federal University \\ Krasnoyarsk, RUSSIAN FEDERATION
}

\begin{abstract}
The method of obtaining the private analytical solution of system of the nonlinear differential equations in private derivatives for calculation the stress state thin-walled beams with rectangular cross section is offered. With use of the semi-return method of SaintVenant the analytical solution which will be agreed with known to the expressions received on dependences of the theory of plates and shells is constructed.

The comparative analysis of results received by finite elements method in Ansys and the offered method showed good convergence, allowed to reveal features of a stress state of beams at pure bend, and also to specify scopes of various types of finite elements.
\end{abstract}

AMS Subject Classification: $60 \mathrm{Kxx}, 81 \mathrm{Sxx}$

Key Words: beam, not axisymmetric cross section, thin-walled elements, plate, stress and deformed state, partial differential equation, method, semi-return method of Saint-Venant, analytical solution

\section{Introduction}

Thin-walled beams with not axisymmetric cross section, and also the compound farm designs based on them, have wide circulation in the most various branches: general and space mechanical engineering, aircraft industry, construction, etc.

Received: $\quad$ December 27, 2016

Revised: $\quad$ January 29, 2017

Published: February 28, 2017

(C) 2017 Academic Publications, Ltd. url: www.acadpubl.eu

$\S_{\text {Correspondence author }}$ 
The existing calculation methods based on hypotheses of the theory of beams [1] and [2] allow to estimate work of all design in general, but at the same time there is no opportunity to consider the stress-deformed state in local areas of thin-walled cross section of a beam in which there is shear stresses causing a deplanation of cross section. Deplanation of cross section depends also on other factors: lengths of a thin-walled beam, thickness of his wall, conditions of loading, fixing, etc.

Use of the simplified models [1] and [2] in the form of the one-dimensional beams having equivalent geometrical and inertial characteristics leads to their very rough model which doesn't allow to create products with the minimum mass-dimensional parameters and the best functional and operational characteristics.

The simplest solution of the accounting of thin-walled cross section is use when calculating such designs of the theory of beams with addition of empirical correction coefficients [3]. However, the results received at the same time strongly depend on the choice of values of these correction coefficients which in reference books are given depending on a combination of certain sizes of cross section of a beam. The choice of intermediate values will be uncertain as at these intervals there is a strong nonlinearity.

The calculation methods created on the basis of fundamental works of $\mathrm{V}$. Z. Vlasov [4], A.R. Rzhanitsyna [5], D. V. Bychkov [6], are development of the theory of beams for a case of thin-walled with axisymmetric cross section. The main achievement of these works is assessment and the accounting of a deplanation of thin-walled cross section in case of action of big shear stress at the constrained torsion or joint action of a bend and torsion. Shortcomings of such approach follow from the initial one-dimensional settlement scheme of a design (beam) chosen by authors [4], [5] and [6] therefore the stress-deformed state of a thin-walled beam in general is estimated, without an opportunity to define his local state.

Operating conditions of precision rod designs, such as the wave guides of the antenna-feeder system representing thin-walled beams with rectangular cross section demand calculation methods, the stress-deformed state allowing to define with high precision in general and in any local area.

It is possible to fulfill the requirements stated above, having only passed when modeling thin-walled beams from their one-dimensional models (beams, beams) to two-dimensional settlement schemes (plates, shells) with the corresponding dependences of the theory of plates and shells [7] and [8].

Calculation of a thin-walled beam with not axisymmetric cross section with use of the theory of shells is complicated by existence of folds (a plate at right 
angle), the functions of radius of curvature of a shell leading to a gap to which conditions on smoothness and a continuity are shown [7]. As a result, it is difficult to describe the stress-deformed state of a thin-walled beam in general by means of one system of the differential equations of the theory of shells. Division of geometry of an initial design into separate elements in the form of plates and shells can be an exit from this situation. However, the known works in this area, for example [9], consider only axisymmetric compound designs for which calculation authors use numerical solutions on the computer.

It is offered to represent also geometry of thin-walled beams with not axisymmetric section system from separate elements (plates, shells) connected among themselves, for each of which the subsystem of the differential equations is under construction. In the case under consideration the thin-walled beam with rectangular cross section is modeled by system from four plates connected at right angle (Fig.1).

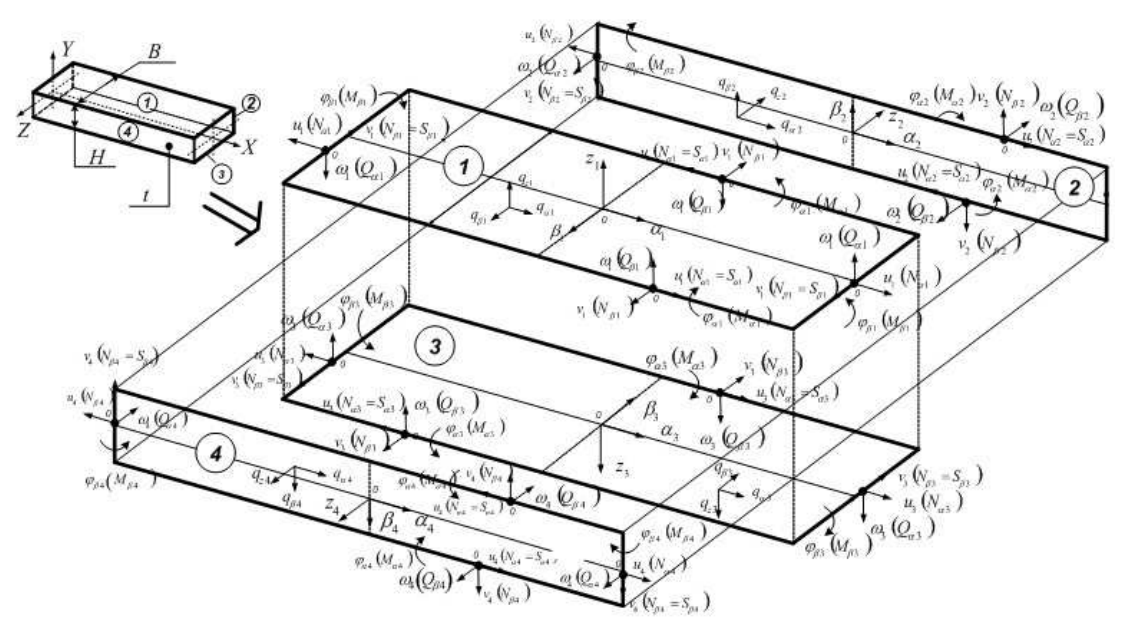

Figure 1: Shell model of thin-walled beam with rectangular cross section

For each of plates the subsystem of the partial differential equations in nonlinear statement $[8,10]$ which set for a thin-walled beam, generally the accounting of dynamic and temperature influences, will have an appearance is 
used:

$$
\left.\begin{array}{r}
\nabla^{4} \phi_{i}=E \cdot\left[\left(\frac{\partial^{2} \omega_{i}}{\partial \alpha_{i} \partial \beta_{i}}\right)^{2}-\frac{\partial^{2} \omega_{i}}{\partial \alpha_{i}^{2}} \cdot \frac{\partial^{2} \omega_{i}}{\partial \beta_{i}^{2}}\right]+ \\
+E \alpha \cdot \nabla^{2}\left(T_{i}\left(\alpha_{i}, \beta_{i}\right)-T_{0 i}\left(\alpha_{i}, \beta_{i}\right)\right) ; \\
\nabla^{4} \omega_{i}=\frac{t}{D}\left[\frac{\partial^{2} \phi_{i}}{\partial \beta_{i}^{2}} \cdot \frac{\partial^{2} \omega_{i}}{\partial \alpha_{i}^{2}}-2 \frac{\partial^{2} \phi_{i}}{\partial \alpha_{i} \partial \beta_{i}} \cdot \frac{\partial^{2} \omega_{i}}{\partial \alpha_{i} \partial \beta_{i}}+\right. \\
\left.+\frac{\partial^{2} \phi_{i}}{\partial \alpha_{i}^{2}} \frac{\partial^{2} \omega_{i}}{\partial \beta_{i}^{2}}-q_{\alpha i} \frac{\partial \omega_{i}}{\partial \alpha_{i}}-q_{\beta i} \frac{\partial \omega_{i}}{\partial \beta_{i}}+q_{Z i}-\rho t \frac{\partial^{2} \omega_{i}}{\partial \tau^{2}}\right] .
\end{array}\right\}
$$

where $i=1,2,3,4$ - numbers of plates;

$\nabla^{2}=\frac{\partial^{2}}{\partial \alpha_{i}^{2}}+2 \frac{\partial^{2}}{\partial \alpha_{i} \partial \beta_{i}}+\frac{\partial^{2}}{\partial \beta_{i}^{2}} ; \nabla^{4}=\frac{\partial^{4}}{\partial \alpha_{i}^{4}}+2 \frac{\partial^{4}}{\partial \alpha_{i}^{2} \partial \beta_{i}^{2}}+\frac{\partial^{4}}{\partial \beta_{i}^{4}} ;$

$\omega_{i}=\omega_{i}\left(\alpha_{i}, \beta_{i}\right)$ - function of deflections for the ith plate;

$\phi_{i}=\phi_{i}\left(\alpha_{i}, \beta_{i}\right)-$ stress function for the ith plate;

$q_{\alpha i}, q_{\beta i}, q_{Z i}$ - components of surface loading for the ith plate;

$E$ - Young's modulus; $\alpha$-thermal expansion coefficient; $\tau$ - time;

$t$ - thickness of plates; $\rho$ - density;

$T_{0 i}\left(\alpha_{i}, \beta_{i}\right)$ - initial temperature field for the $i$-th plate;

$T_{i}\left(\alpha_{i}, \beta_{i}\right)$ - temperature field for the ith plate when heating.

Boundary conditions on lateral faces of plates will be defined by required unknown functions $\phi_{i}(\alpha, \beta)$ and $\omega_{i}(\alpha, \beta)$ in forms:

$$
\begin{gathered}
\left.t \cdot \frac{\partial^{2} \phi_{i+1}}{\partial \alpha_{i+1}^{2}}\right|_{\beta_{i+1}=b_{i}+1}=-\left.D_{i}\left(\frac{\partial^{3} \omega_{i}}{\partial \beta_{i}^{3}}+\frac{\partial^{3} \omega_{i}}{\partial \beta_{i} \partial \alpha_{i}^{2}}\right)\right|_{\beta_{i}=-b_{i}} ; \\
\left.\frac{\partial^{2} \phi_{i+1}}{\partial \alpha_{i+1} \partial \beta_{i+1}}\right|_{\beta_{i+1}=b_{i+1}}=\left.\frac{\partial^{2} \phi_{i}}{\partial \alpha_{i} \partial \beta_{i}}\right|_{\beta_{i}=-b_{i}} ; \\
-\left.D_{i}\left(\frac{\partial^{3} \omega_{i+1}}{\partial \beta_{i+1}^{3}}+\frac{\partial^{3} \omega_{i+1}}{\partial \beta_{i+1} \partial \alpha_{i+1}^{2}}\right)\right|_{\beta_{i+1}=b_{i+1}}=\left.t \cdot \frac{\partial^{2} \phi_{i}}{\partial \alpha_{i}^{2}}\right|_{\beta_{i}=-b_{i}} ; \\
\frac{\partial^{2} \omega_{i+1}}{\partial \alpha_{i+1}^{2}}+\left.\mu \frac{\partial^{2} \omega_{i+1}}{\partial \beta_{i+1}^{2}}\right|_{\beta_{i+1}=-b_{i+1}}=\frac{\partial^{2} \omega_{i}}{\partial \alpha_{i}^{2}}+\left.\mu \frac{\partial^{2} \omega_{i}}{\partial \beta_{i}^{2}}\right|_{\beta_{i}=-b_{i}} ;
\end{gathered}
$$

where $D_{i}$ - cylindrical rigidity of the $i$-th plate.

$b_{i}, b_{i+1}$ - breadth of the $i$-th and $(i+1)$-th plate, respectively.

Boundary conditions at end faces of each plate determine conditions of loading and fixing of a thin-walled beam in general by his edges. 
The system of the nonlinear partial differential equations (1.1), together with boundary conditions (1.2), represents the general problem definition that allows at its solution to use wide variability of various approaches and techniques in relation to requirements of specifically solved task, both in relation to spatial thin-walled designs with not axisymmetric cross section and the corresponding operational conditions.

The offered approach can be used for modeling of thin-walled cores of any not axisymmetric section: two-Tauri, channel, $Z$-profile, etc.

However, now there is no common analytical solution even for one subsystem of a separate plate in system (1.1) on what pointed still to S. P. Tymoshenko [8] and the more so for four such subsystems which joint solution describes the stress state of a thin-walled beam with rectangular cross section. Thus, methods of modeling and creation of the analytical solution of thin-walled cores with not axisymmetric form of cross section on the basis of the theory of shells and plates are insufficiently developed now.

\section{Problem Definition of a Pure Bend}

We will consider a simple case of a stress state of a straight part of a beam: pure bend concerning an axis $Z$ in global system of coordinates under the influence of the bending moment (fig. 2, a) in the plane of the minimum rigidity. At isothermal process of loading of a straight part of a beam in the first equation of system (1.1), the temperature component will be equal to zero: $E \alpha \cdot \nabla^{2}\left(T_{i}\left(\alpha_{i}, \beta_{i}\right)-T_{0 i}\left(\alpha_{i}, \beta_{i}\right)\right)=0$, and surface load is also equal to zero: $q_{\alpha i}=q_{\beta i}=q_{Z i}=0$.

However, obtaining the analytical solution of system (1.1) is still complicated by coherence of the differential equations among themselves and nonlinearity of system. For the purpose of simplification of the solution, we will consider deformation of cross section of a core at a bend. If the straight part of a beam is affected by the bending $M_{Z}$ moment, cross section will be deformed changing the sizes and consistently taking the forms 1-4 (fig. 2, b). Thus forms 3, 4 will already correspond to loss of stability of all separate plates making geometry of cross section [11].

In relation to the considered case it means that at action of the bending moment and an assumption about a trifle of deformations, each of plates 1-4 will be bent only in one of the planes of the local system of coordinates, being left in other coordinate planes without change of a form that will be expressed 
as a condition:

$$
\theta_{\beta_{i}}=\frac{\partial \omega_{i}}{\partial \beta_{i}}=0
$$

Substitution of a condition (2.1) in system (1.1) leads to simplification and linearization of her equations and boundary conditions which will take a form:

$$
\begin{gathered}
\nabla^{4} \phi_{i}=0 ; \nabla^{4} \omega_{i}=0 . \\
\left.\frac{\partial^{2} \phi_{i+1}}{\partial \alpha_{i+1}^{2}}\right|_{\beta_{i+1}=b_{i+1}}=0 ;\left.\frac{\partial^{2} \phi_{i+1}}{\partial \alpha_{i+1} \partial \beta_{i+1}}\right|_{\beta_{i+1}=b_{i+1}}=\left.\frac{\partial^{2} \phi_{i}}{\partial \alpha_{i} \partial \beta_{i}}\right|_{\beta_{i}=-b_{i}} ; \\
\left.\frac{\partial^{2} \phi_{i}}{\partial \alpha_{i}^{2}}\right|_{\beta_{i}=-b_{i}}=0 ;\left.\frac{\partial^{2} \omega_{i+1}}{\partial \alpha_{i+1}^{2}}\right|_{\beta_{i+1}=-b_{i+1}}=\left.\frac{\partial^{2} \omega_{i}}{\partial \alpha_{i}^{2} \partial \beta_{i}}\right|_{\beta_{i}=-b_{i}} .
\end{gathered}
$$

From the geometrical point of view, the condition (2.1) is equivalent only to one requirement - the straight lines forming a form of cross section of a thin-walled beam under loading have to remain straight lines that considerably generalizes the offered assumption.

\section{Method of Obtaining the Analytical Solution}

Functions also define the stress-deformed state of each $i$-th of a plate separately. Combination of these solutions, according to the principle of superposition, for each separate plate will allow to define finally the stress-deformed state of a thin-walled beam in general. The analytical solution for functions and for a case of a pure bend (Fig. 2, a) we will get taking into account features a job of each $i$-y of a plate in its structure:

- side plates 2 and 4 test a pure bend in the plane;

- plates 1 and 3 are exposed to stretching and compression respectively in combination with a bend on the curve formed by the deformed edges of plates 2 and 4 .
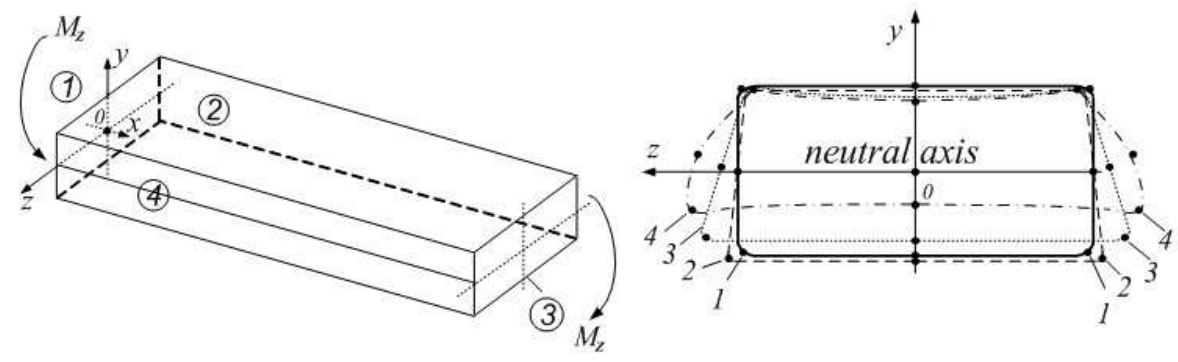


\section{a) b)}

Figure 2: Analytical solution for functions and for a case of a pure bend

Let's use the semi-return method of Saint-Venant $[12,13,14,15]$ with direct determination of stress (function of Airy) and movements (deflections) for each plate which will allow to receive for them private solutions which combination will be the analytical solution of the considered task in general.

\subsection{Functions of Stresses}

According to the semi-return method of Saint-Venant, we will receive expression for functions of Erie on the basis of the known approximate approach as which we will make the solution according to the theory of beams [1]. Then the diagramm of distribution of normal stress in plates of a thin-walled beam at a bend will be defined according to Navier [1] formula (Fig. 3).

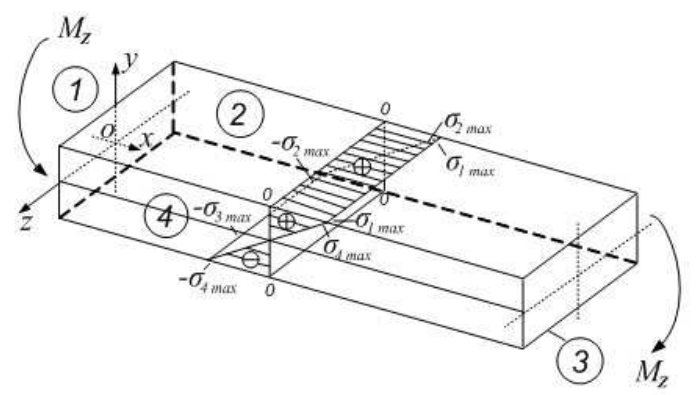

Figure 3: The general stress state of the beam at a bend

Expressions for functions of Erie it agrees [7, 8], will take a form:

$$
\left.\begin{array}{c}
\phi_{1}\left(\alpha_{1}, \beta_{1}\right)=\beta_{1}^{2} \cdot \frac{M_{Z}}{J_{Z}} \cdot \frac{h^{\prime}}{4} ; \phi_{2}\left(\alpha_{2}, \beta_{2}\right)=\frac{M_{Z}}{J_{Z}} \cdot \frac{\beta_{2}^{3}}{6} ; \\
\phi_{3}\left(\alpha_{3}, \beta_{3}\right)=-\beta_{3}^{2} \cdot \frac{M_{Z}}{J_{Z}} \cdot \frac{h^{\prime}}{4} ; \phi_{4}\left(\alpha_{4}, \beta_{4}\right)=-\frac{M_{Z}}{J_{Z}} \cdot \frac{\beta_{4}^{3}}{6} .
\end{array}\right\}
$$

where: $J_{Z i, j}$ - the axial moment of the cross section (fig. 3).

Analyzing dependences (3.1) it is visible that they determine only a constant component of stress in plates 1 and 3 by their thickness, corresponding to stresses in their median surfaces. Therefore, the received solution (3.1) for plates 1 and 3 needs to be added with the expressions considering flexural stress from their non-central stretching compression.

The deflection of plates 1 and 3 will be defined generally by deformations of their lateral faces [16] on which there are power and kinematic interactions 
of the plates interfaced to them 2 and 4. For this purpose in system (2.2) it is necessary to receive functions of deflections of plates.

\subsection{Functions of Deflections}

According to the accepted assumption (2.1), plates 2 and 4 will be bent everyone in the plane, therefore, their deflections from the plane are equal to zero:

$$
\omega_{2}\left(\alpha_{2}, \beta_{2}\right)=\omega_{4}\left(\alpha_{4}, \beta_{4}\right)=0 .
$$

The deformed condition of plates 1 and 3, we will define on the basis of the semi-return method of Saint-Venant with direct definition of movements. For this purpose from the main dependences of the theory of elasticity [15] we will receive expressions for functions of movement of lateral faces of plates 2 and 4 by which deflections of borders of plates 1 and 3 in places of their connection will be defined (Fig. 4).

$$
\omega_{1}=\nu_{2}=\frac{M_{Z}}{2 \cdot E J_{Z}}\left[\alpha_{2}^{2}+\mu \cdot\left(\beta_{2}^{2}-z_{2}^{2}\right)\right], \omega_{3}=\nu_{4}=\frac{M_{Z}}{2 \cdot E J_{Z}}\left[\alpha_{4}^{2}+\mu \cdot\left(\beta_{4}^{2}-z_{4}^{2}\right)\right] .
$$

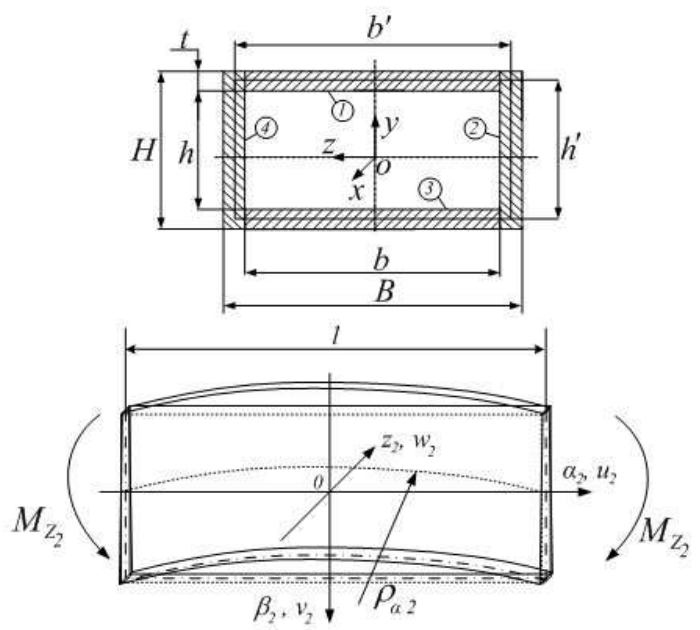

a) deformation of a plate in the plane b) deformation of cross section

Figure 4: The deformed state of a plate 2

Let's substitute $\beta_{2}=-\frac{h^{\prime}}{2}$ and $z_{2}=0$ in (3.3) coordinates and for lateral face of a plate 2 (figs. 4 ), then tangential movements of a side side of a plate 2 will define required function of deflections $\omega_{1}(\alpha, \beta)$ of the plate attached to her 1 :

$$
\omega_{1}=\nu_{2}=\frac{h^{\prime}}{2}-\frac{M_{2}}{2 \cdot E J_{2}}\left[\alpha_{1}^{2}+\mu \cdot \frac{h^{\prime 2}}{4}\right] .
$$




\subsection{The Full Analytical Solution at a Pure Bend}

Using the offered approach, we will receive the dependences similar (3.4), in connections between other plates of a thin-walled beam. Then after association of expressions for functions $\phi_{i}\left(\alpha_{i}, \beta_{i}\right)$ and $\omega_{i}\left(\alpha_{i}, \beta_{i}\right)$ we will also receive the full analytical solution of system (2.2) at a pure bend of a thin-walled beam

$$
\left.\begin{array}{r}
\phi_{1}=\beta_{1}^{2} \cdot \frac{M_{Z}}{J_{Z}} \cdot \frac{h^{\prime}}{4} ; \omega_{1}=\frac{h^{\prime}}{2}- \\
-\frac{M_{Z}}{2 \cdot E J_{Z}}\left(\alpha_{1}^{2}+\mu \cdot \frac{h^{\prime 2}}{4}\right) ; \phi_{2}=\frac{M_{Z}}{J_{Z}} \cdot \frac{\beta_{2}^{3}}{6} ; \omega_{2}=0 ; \\
\phi_{3}=-\beta_{3}^{2} \cdot \frac{M_{Z}}{J_{Z}} \cdot \frac{h^{\prime}}{4} ; \omega_{3}=-\frac{h^{\prime}}{2}+ \\
+\frac{M_{Z}}{2 \cdot E J_{Z}}\left(\alpha_{3}^{2}+\mu \cdot \frac{h^{\prime 2}}{4}\right) ; \phi_{4}=-\frac{M_{Z}}{J_{Z}} \cdot \frac{\beta_{4}^{3}}{6} ; \omega_{4}=0 .
\end{array}\right\}
$$

Using expressions (3.5), it is possible to determine stress and deformations in any point $i$-x plates $(i=1,2,3,4)$ making a thin-walled beam at his pure bend.

\section{Stress at a Pure Bend}

The normal stress operating in $i$-y to a plate will be determined by dependences $[15]$ :

$$
\begin{gathered}
\sigma_{\alpha i}=\sigma_{\phi i}+\sigma_{\omega i}=\frac{\partial^{2} \phi_{i}}{\partial \beta_{i}^{2}}-\frac{E \cdot z_{i}}{1-\mu^{2}}\left(\frac{\partial^{2} \omega_{i}}{\partial \alpha_{i}^{2}}+\mu \frac{\partial^{2} \omega_{i}}{\partial \beta_{i}^{2}}\right) \\
\sigma_{\beta i}=\sigma_{\phi i}+\sigma_{\omega i}=\frac{\partial^{2} \phi_{i}}{\partial \alpha_{i}^{2}}-\frac{E \cdot z_{i}}{1-\mu^{2}}\left(\frac{\partial^{2} \omega_{i}}{\partial \beta_{i}^{2}}+\mu \frac{\partial^{2} \omega_{i}}{\partial \alpha_{i}^{2}}\right) . \\
\sigma_{z i}=0 .
\end{gathered}
$$

According to dependences $(4.1,4.2)$, the largest normal stress will arise in the longitudinal directions $\alpha_{i}(i=1,2,3,4)$ of all plates of a thin-walled beam.

\subsection{Maximum Normal Stress}

According to the received private analytical solution (3.5) and dependence (4.1), diagram of the specified distribution of normal stresses $\sigma_{\alpha i}$ for a thin-walled 
beam are constructed (figs. 5), which maximum values are equal:

$$
\begin{gathered}
\sigma_{1 \max / \min }=\frac{M_{Z}}{J_{Z}} \cdot\left(\frac{h^{\prime}}{2} \pm \frac{t}{2} \cdot \frac{1}{\left(1-\mu^{2}\right)}\right) ; \\
\sigma_{2 \max }=\frac{M_{Z}}{J_{Z}} \cdot \frac{H}{2} ; \sigma_{2 \min }=\frac{M_{Z}}{J_{Z}} \cdot \frac{h}{2} ; \sigma_{1 m}=\sigma_{2 m}=\frac{M_{Z}}{J_{Z}} \cdot \frac{h^{\prime}}{2} .
\end{gathered}
$$

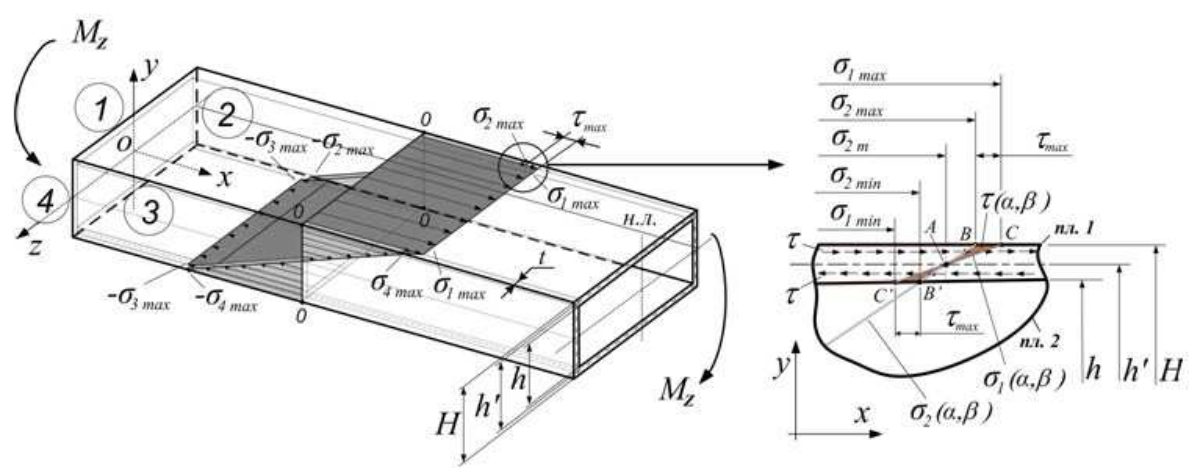

a) refined stress state b) stress state in a junction of plates 1 and 2

Figure 5: General and local refined stress state of straight part of beam at a bend

Shows comparison of expression for the maximum stress $\sigma_{1 \max }(4.3)$ and Navier [1] formula that they differ only in a "flexural" component of stress and under a condition

$$
\left(1-\mu^{2}\right) \approx 1,
$$

that is without squeezing of fibers in plates 1,3 coincide:

$$
\sigma_{1 \max }=-\sigma_{3 \max }=\frac{M_{Z}}{J_{Z}} \cdot\left(\frac{h^{\prime}}{2}+\frac{t}{2}\right)=\frac{M_{Z}}{J_{Z}} \cdot \frac{H}{2} .
$$

Not the accounting of squeezing of longitudinal fibers is among themselves used as one of fundamental hypotheses of the theory of beams at their bend [1] and doesn't lead to big errors in calculations at the small sizes of elements in the cross directions. For thin-walled elements of a thin-walled beam which are bent from the plane (a plate 1,3 on figs. 2), the specified hypothesis is unacceptable.

\subsection{Maximum Shear Stress}

Analysis shear normal stress (figs. 5) for plates 1 and 2 in places of their connection on lateral faces shows existence in values of stress of sharp jumps 
which cause emergence of shear stress. The similar situation will arise also in junctions of lateral faces of other plates 2-3, 3-4 and 4-1.

Values of shear stress change under the linear law (figs. 5), reaching the maximum values on external and internal surfaces of a plate 1 will also be equal:

$$
\begin{aligned}
\tau_{\max }=\sigma_{1 \max }-\sigma_{2 \max }=\frac{M_{Z}}{J_{Z}}\left(\frac{h^{\prime}}{2}+\frac{t}{2} \cdot \frac{1}{\left(1-\mu^{2}\right)}\right)-\frac{M_{Z}}{J_{Z}} & \cdot \frac{H}{2}= \\
= & \frac{t}{2} \cdot \frac{\mu^{2}}{1-\mu^{2}}
\end{aligned}
$$

Emergence of shear stress at a bend of the thin-walled beam having rectangular cross section, obviously is caused by geometrical heterogeneity of his form. Shear stress works only in junctions of plates 1-2, 2-3, 3-4, 4-1 and has local self-balanced character, without influencing the general the stress-deformed state.

However, in certain cases, shear stress can have significant effect on operability of designs. For example, at connection of thin-walled plates among themselves welded or solder connections which are loaded with dynamic and temperature cyclic loadings.

\section{Results of Comparative Calculations and their Analysis}

For verification of the developed technique comparative calculations for Navier's formula and are carried out to Ansys for thin-walled beams with different sizes of their cross sections: $5.5 \times 11 \times 1.2 ; 10 \times 61 \times 2 ; 6.5 \times 13 \times 1.2 ; 8 \times 16 \times 1.2$; $9.5 \times 19 \times 1.2 ; 12.6 \times 28.5 \times 1.2 ; 15 \times 35 \times 1.2 ; 25 \times 58 \times 2 ; 50 \times 116 \times 2$ and $100 \times 232 \times 2$.

For receiving the most uniform field of stress for each standard size of cross section the required length of a thin-walled beam, his way of fixing and loading were defined.

Calculated by the offered technique of value of the maximum normal (4.3) and tangent (4.7) stress were compared to results of the calculations received on Navier (3.11) formula and method of finite elements in Ansys for various types of the finite elements (FE) with linear and square functions of a form:

1) frame FE: Beam4, Beam188, Beam189;

1) shell FE: linear Shell181 and square Shell281;

2) solid-state FKE: linear Solid185 and square Solid98.

The number of finite elements was defined by a condition of achievement of the greatest possible uniform field of stress and has made: for rod model about 
one thousand KE, for shell model 7-9 thousand FE and for solid-state model of 40-60 thousand KE.

\subsection{Normal Stress}

Results of calculation of the maximum flexural normal stress in plates of the beams by all compared techniques are shown on fig. 6 in relative values. At assessment of relative value of result (in \%) as basic value of the maximum stress the size calculated according to the technique offered by us and dependence (4.3) is accepted.

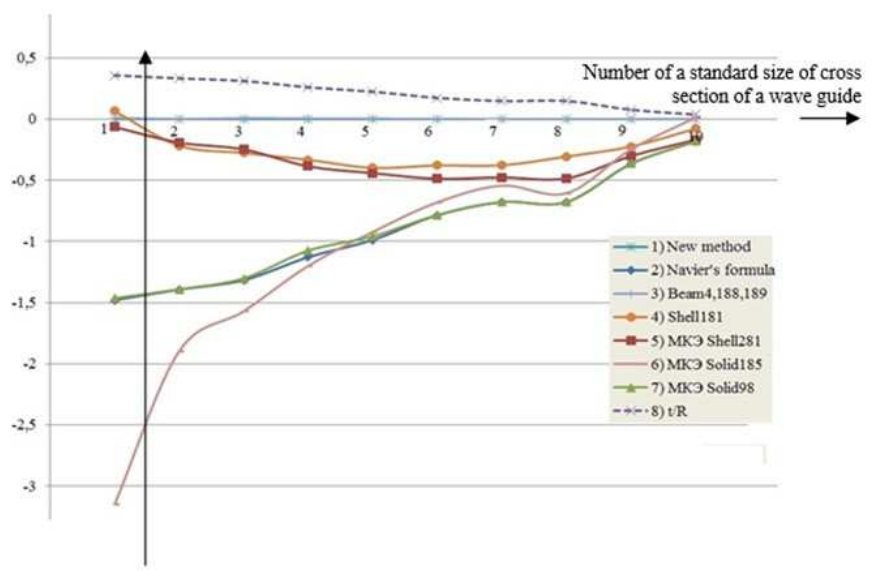

Figure 6: Comparison of relative values (\%) maximum normal stresses

Thus, schedules on fig. 6 show a deviation of values of the maximum normal stress by the considered and analyzed calculation procedures (in \%) concerning the results received by the offered technique (4.3) for the considered standard sizes (No. 1-10) of cross section of beams.

The executed calculations have shown that the analytical solution (4.3) differs from results, the received other methods, for $0.1-3.1 \%$.

\subsection{Shear Stress}

Values of the maximum shear stress calculated by a formula (4.7) for the considered sizes of sections of beams make only $0.18-1.5 \%$ of the level of the maximum normal stress (4.3). At increase of the characteristic relation (4.2) the level of the maximum shear stress decreases (Fig. 7). 


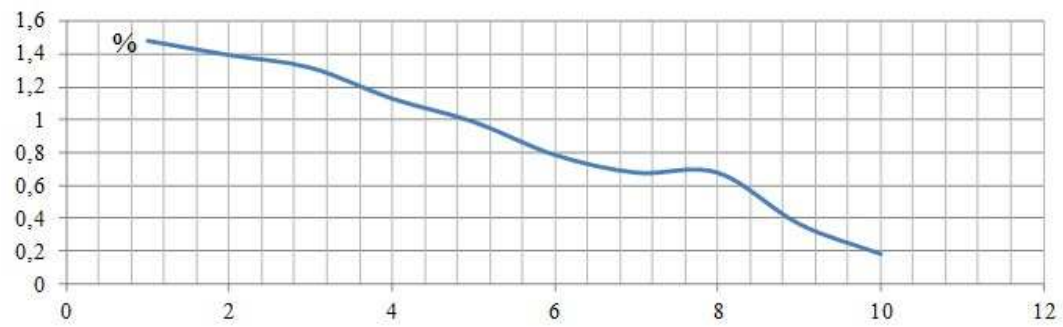

Figure 7: Relative values of the maximum shear stresses

Other considered methods of calculations (Navier's formula and FEM for various types of finite elements) haven't recorded emergence of shear stress in junctions of plates.

\subsection{Features of the FEM-Solution}

The analysis of the results shown on figs. 6, allows to estimate features of numerical solutions:

1) Results of calculations of flexural normal stress for all FE Beam4, Beam188 and Beam189 frame types (a curve 3) are completely identical each other, and are comparable to calculations for Navier's formula (a curve 2) as frame finite elements when determining flexural normal stress use Navier's formula [17] and [18].

2) Results of calculations with solid-state Solid185 and Solid98 FE (curves 6,7 ) have almost completely coincided with the results of calculations received on Navier's formula as knots of solid-state FE have only forward degrees of freedom don't consider a movement trajectory, and in the received analytical solution the amendment goes at the expense of the accounting of curvature of plates.

3) When using the shell of FE of the Shell181 types (a curve 4) and Shell182 (a curve 5) settlement dependences are based on hypotheses of the theory of plates and shells $[7,17,18]$. It allows to consider change curvature thin-walled elements (plates) that causes the essential growth of the maximum normal stress in comparison with calculations on Navier's formula and FEM with solid-state finite elements.

Results of calculations of the maximum normal stress in plates of a thinwalled beam by the offered technique were closest to numerical results of FEM at application the shell of FE and differ no more than for $0.5 \%$. 


\section{Conclusion}

The way of modeling of the intense deformed condition of thin-walled beams of rectangular cross section with use of the theory of plates and shells is offered.

The system of the differential equations in private derivatives for the general case of loading of a thin-walled beam of rectangular cross section in nonlinear statement is received and complexity of her solution is shown.

With use of the semi-return method of Saint-Venant the private analytical solution of system of the differential equations for a case of a pure bend of a thin-walled beam in linear statement is received.

The solution of a task in idle time analytical to a form which has allowed to specify expression for Navier's formula when determining the maximum values of normal stress is received, and also to reveal local scopes of the shear stress at a pure bend caused by heterogeneity of a form of cross section of a thin-walled beam and to estimate their values.

The comparative analysis of results of calculations in relation to beams received by a numerical method of finite elements and the offered technique, has shown their good convergence, distinction has made 0.1-3.1\%, and has allowed to reveal features of application of various types of finite elements.

The offered approach and technique of obtaining the analytical solution can be used for the analysis of the stress-deformed state of thin-walled beams of any other form of thin-walled not axisymmetric cross section: two-Tauri, channel, $Z$-profile, etc.

\section{Acknowledgments}

The reported study was funded by Russian Foundation for Basic Research, Government of Krasnoyarsk Territory, Krasnoyarsk Region Science and Technology Support Fund to the research project No 16-48-242110.

\section{References}

[1] V.I. Feodosyev, Strength of materials, Moscow, MGTU (1999).

[2] V.I. Feodosyev, Selected problems and questions on strength of materials, Moscow, Nauka (1967).

[3] L.V. Agamirov, Strength of materials, Moscow, Astrel (2003).

[4] V.Z. Vlasov, Selected Works, Vol. 2: Thin elastic rods. Principles of general technical theory of shells, Moscow, USSR AS Press (1963). 
[5] A.R. Rzhanicyn, Structural mechanics, Moscow, Higher School (1982).

[6] D.V. Bychkov, Structural Mechanics core thin-walled structures, Moscow, Gosstrojizdat (1962).

[7] V.V. Novozhilov, K.F. Chernykh, E.I. Mikhaylovskiy, Linear theory of thin shells, SaintPetersburg, Saint-Petersburg University Press (2010).

[8] S.P. Timoshenko, S. Voynovskiy-Kriger, Plates and shells, Moscow, URSS Press (2009).

[9] V.I. Myachenkov, I.V. Grigoriev, Calculation of composite shell designs on a computer, Moscow, Mashinostroenie (1981).

[10] A.S. Volmir, Flexible plates and shells, Moscow (1956).

[11] D. Kecman, Bending collapse of rectangular and square section tubes. International Journal of Mechanical Sciences, 25 (1983), 623-636.

[12] P.F. Papkovich, Theory of elasticity, Moscow, GIOP Publ. (1939).

[13] S.P. Timoshenko, J. Gudyer, Theory of elasticity, Moscow, Nauka (1979).

[14] V. Parton, P.I. Perlin, Methods of Mathematical Theory of Elasticity, Moscow, Nauka (1981).

[15] A.V. Aleksandrov, Fundamentals of the theory of elasticity and plasticity, Moscow, (1990).

[16] P.N. Silchenko, I.V. Kudryavtsev, M.M. Mihnev, O.B. Gocelyuk, Some approaches to preparation of differential equations solutions for the element waveguide channel spacecraft, Vestnik NIYAU MIFI, 4 (2015), 19-24.

[17] Ansys Help Release 14.5, Theory Reference, Element Library (2014).

[18] M.P. Galanin, Methods of mathematical models numerical analysis, Moscow, Bauman MGTU (2012). 
